\title{
Production of Prompt Photons
}

\author{
Edmond L. Berger \\ High Energy Physics Division, Argonne National Laboratory, Argonne, IL, 60439
}

\begin{abstract}
After a brief review of the production dynamics of prompt single photons in hadron collisions, I summarize a new QCD calculation of the transverse momentum distribution of continuum prompt photon pairs produced by QCD subprocesses, including all-orders soft-gluon resummation valid at next-to-next-to-leading logarithmic accuracy. Resummation is necessary to obtain reliable predictions, as well as good agreement with data from the Fermilab Tevatron, in the range of transverse momentum where the cross section is largest. Predictions are made for the Large Hadron Collider where the QCD diphoton continuum is shown to have a softer spectrum in transverse momentum than the Higgs boson signal.
\end{abstract}

Keywords: QCD, Photons, Tevatron, LHC, Higgs

PACS: $12.15 . J i, 12.38 \mathrm{Cy}, 13.85 . \mathrm{Qk}$

\section{INTRODUCTION}

The study of prompt photons carrying large values of transverse momentum has a history almost as old as quantum chromodynamics (QCD). It ranks with deep-inelastic-lepton scattering, massive-lepton-pair production (the 'Drell-Yan process'), and jet production as an important probe of short-distance hadron dynamics ${ }^{1}$. The appeal of prompt photons is that they are point-like, colorless probes of the dynamics of quarks and gluons, ones that escape unscathed through the colored medium of the high-energy collision. One motivation, in spin-dependent and spin-averaged scattering, is the access that prompt photons offer to the spin-dependent and spin-averaged gluon densities of hadrons. One of the two leading-order partonic direct subprocesses feeds directly from the gluon parton density through the gluon 'Compton' subprocess, $q g \rightarrow \gamma q$.

In reality, the promise and naive simplicity of prompt photons are compromised by two issues: fragmentation contributions and photon isolation. Fragmentation is a longdistance process in which a hard photon brems off a final-state quark (or gluon). The photon emerges as part of a jet if the opening angle between the quark and photon is too small. This 'showering' process must be parameterized by a non-perturbative singlephoton fragmentation function $D_{\gamma}\left(z, \mu_{f}\right)$. Variable $z$ is the fraction of the parent parton's momentum that the photon retains, and fragmentation scale $\mu_{f}$ serves as a boundary in momentum $\mu$ space between the long-distance dynamics included in $D_{\gamma}\left(z, \mu_{f}\right)$ and the short-distance next-to-leading order dynamics in the region $\mu>\mu_{f}$ (i.e., large angle separation between the final photon and remnants of the fragmenting parton). Note that a new variable $\mu_{f}$ enters the mix along with the usual renormalization and factorization

${ }^{1}$ A photon is said to be prompt if it cannot be said to originate from the decay of a hadron, such as a $\pi^{0}$ or $\eta$, itself produced with large transverse momentum. 
scales $\mu_{R}$ and $\mu_{F}$.

A theory calculation is most reliable for an inclusive observable since infra-red and collinear singularities can be shown to cancel between the real-emission and loop diagrams in an inclusive calculation that is carried beyond the leading order in perturbation theory. However, Tevatron and Large Hadron Collider (LHC) experiments measure isolated photons in which part of the real-emission final-state phase space is excluded, resulting in incomplete cancellation of singularities in an analytic calculation [1]. Isolation is required since, otherwise, the prompt photon signal would be overwhelmed by secondary $\gamma$ 's from hadron decays, and it is hard to discern a prompt $\gamma$ if it is buried in a jet (unless $z$ is very large). In isolation procedures applied by the experiments, the energy of the hadronic remnants is required to be less than a threshold isolation energy $E_{T}^{i s o}$ in a cone $\Delta R=\sqrt{\Delta \eta^{2}+\Delta \varphi^{2}}$ around each photon, with $\Delta \eta$ and $\Delta \varphi$ being the separations of the hadronic remnant(s) from the photon in the plane of pseudo-rapidity $\eta$ and azimuthal angle $\varphi$. The values of $E_{T}^{i s o}$ and $\Delta R$ characterize the measurement, and, in a theory calculation, the size of the fragmentation contributions depends on the assumed values of these parameters ${ }^{2}$. Theoretical representations of isolation are at best approximate. Calculations tend to require Monte Carlo modeling of fragmentation in order to implement experiment-like isolation selections.

Calculations exist of prompt single photon production at next-to-leading order in QCD for both the direct and the fragmentation contributions. One approach is encoded in the Monte Carlo program JETPHOX [2]. An impressive comparison with experiment may be found in the D0 paper [3].

Much more can be written about recent research on inclusive and isolated single photon production, but I turn to production of photon pairs in the remainder of this brief report, summarizing a new calculation of the diphoton cross section in perturbative QCD [4]. The motivation for this study comes, in part, from the fact that a Higgs boson with mass between 115 and $140 \mathrm{GeV}$ may be identified at hadron colliders through its decay into a pair of energetic photons. Theoretical predictions of the QCD continuum 'background' may be of substantial value in aiding search strategies. Moreover, the QCD calculation of continuum photon-pair production is of theoretical interest in its own right, and data from the Tevatron collider [5] offer an opportunity to test results against experiment.

I focus on the transverse momentum $\left(Q_{T}\right)$ distribution of a pair of photons from the QCD continuum and from the Higgs boson signal. This distribution is important because its behavior affects the precision of the determination of the vertex from which the Higgs boson emerges. Greater activity in $Q_{T}$ associated with Higgs boson production allows a more precise determination of the event vertex. The expected shape of $d \sigma / d Q_{T}$ can affect triggering and analysis strategies. Event modeling, kinematic acceptance, and efficiencies all depend on $Q_{T}$. Finally, as is shown here, selections on $Q_{T}$ may be useful to enhance the signal to continuum ratio.

${ }^{2}$ In the case of production of photon pairs, discussed below, the two photons must also be separated in the $\eta-\varphi$ plane by an amount exceeding the approximate angular size $\Delta R_{\gamma \gamma}$ of one calorimeter cell. 


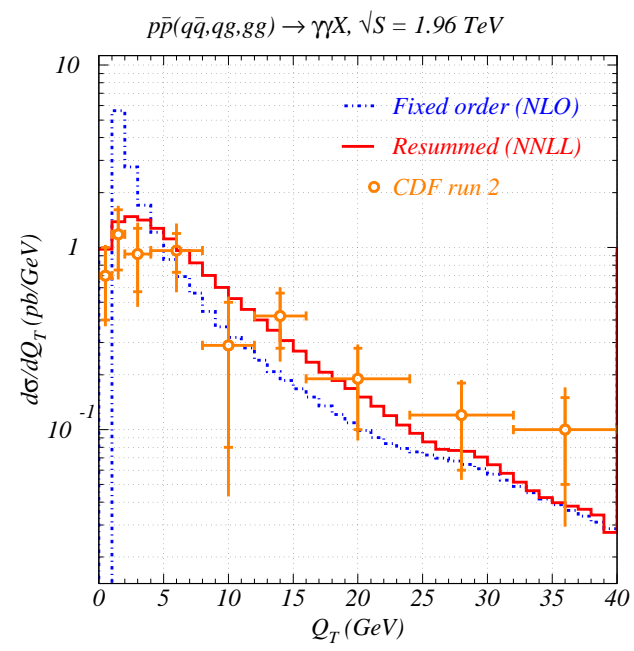

FIGURE 1. Transverse momentum distributions of continuum diphotons for $p \bar{p} \rightarrow \gamma \gamma X$ at $\sqrt{s}=$ $1.96 \mathrm{TeV}$. The dotted histogram shows the result of the next-to-leading order calculation and the solid histogram the result after resummation at NNLL accuracy. The data are from the CDF measurement.

\section{CALCULATION OF THE DIPHOTON CONTINUUM}

Direct production of continuum photon pairs occurs from $q \bar{q}, \stackrel{(-)}{q} g$, and $g g$ scattering. In Ref. [4], next-to-leading order (NLO) perturbative cross sections are included, i.e., cross sections of $\mathscr{O}\left(\alpha_{s}\right)$ in the $q \bar{q}$ and $q g$ channels, and $\mathscr{O}\left(\alpha_{s}^{3}\right)$ in the $g g$ channel. Singular logarithms arise in the NLO cross sections when $Q_{T}$ of the $\gamma \gamma$ pair is much smaller than its invariant mass $Q$. To describe properly the behavior of the $Q_{T}$ distribution in this region $Q_{T}<Q$, where the cross section is greatest, all-orders resummation of initial-state soft and collinear logarithmic contributions is included, based on the Collins-Soper-Sterman (CSS) resummation procedure [6]. The logarithms are (re)summed into a Sudakov exponent (composed of functions $A(\mu)$ and $B(\mu)$ ) and convolutions of the conventional parton densities $f_{a}\left(x, \mu_{F}\right)$ with Wilson coefficient functions $C$. We include the $\mathscr{O}\left(\alpha_{s}^{3}\right)$ expressions for $A(\mu), \mathscr{O}\left(\alpha_{s}^{2}\right)$ expressions for $B(\mu)$, and the $C$-functions at order $\alpha_{s}$, for all subprocesses. These enhancements elevate the accuracy of the resummed prediction to the next-to-next-to-leading-logarithmic (NNLL) level.

\section{COMPARISON WITH TEVATRON DATA}

Our analysis provides the triple-differential cross section $d \sigma / d Q d Q_{T} d \Delta \phi(\Delta \phi$ is the difference of the angles of the two photons in the transverse plane). The calculation is especially pertinent for the transverse momentum distribution in the region $Q_{T} \leq Q$, for fixed values of diphoton mass $Q$. It would be best to compare our multi-differential distribution with experiment, but the collider data tend to be presented in the form of single-differential distributions in $Q, Q_{T}$, and $\Delta \phi$, after integration over the other variables. For calculations appropriate at Tevatron energies, we impose cuts $\left|y_{\gamma}\right|<0.9$ on the rapidity of each photon, and $p_{T}^{\gamma}>p_{T \min }^{\gamma}=14$ (13) GeV on the transverse momentum 
of the harder (softer) photon in each $\gamma \gamma$ pair. We choose $E_{T}^{i s o}=1 \mathrm{GeV}, \Delta R=0.4$, and $\Delta R_{\gamma \gamma}=0.3$.

The $Q_{T}$ distribution is shown in Fig.1 The resummed $Q_{T}$ distribution is well-behaved as $Q_{T} \rightarrow 0$, unlike its fixed-order counterpart which is singular in this limit. The result after NNLL resummation is in good agreement with the absolute rate and overall shape of the data from the Collider Detector at Fermilab (CDF) collaboration at $p \bar{p}$ collision energy $\sqrt{s}=1.96 \mathrm{TeV}$ [5].

In the two highest- $Q_{T}$ bins, there is some evidence of an excess experimental rate, perhaps a 'shoulder' in the data. Our calculations show that most of the shoulder events populate a limited volume of phase space characterized by small $\Delta \phi$ and $Q_{T}>Q$. From a theoretical point of view, when $Q_{T}>Q$, as in the shoulder region, the calculation must be organized in a different way [7] in order to resum contributions arising from the fragmentation of partons into a pair of photons with small invariant mass, a new feature of diphoton production with respect to single photon production. Adequate treatment of the light $\gamma \gamma$ pairs is missing in our calculation and in all other calculations at present. This interesting region warrants further theoretical investigation. Our theoretical treatment is most reliable in the region $Q_{T}<Q$. When this selection is made, the contributions from small $\Delta \phi$ are efficiently suppressed, and dependence on tunable isolation parameters and factorization scales is reduced. After the selection $Q_{T}<Q$, we expect that the large $Q_{T}$ shoulder will disappear in the experimental $Q_{T}$ distribution.

Predicted distributions in the invariant mass $Q$ of the photon pair and the separation $\Delta \phi$ may be found in Ref. [4]. Suggestions are made there for more differential analyses of the Tevatron data that would allow refined tests of the calculation. An important consequence of the resummation formalism is a logarithmic dependence on the diphoton invariant mass $Q$. We encourage the $\mathrm{CDF}$ and $\mathrm{D} 0$ collaborations to verify this predicted broadening of the $Q_{T}$ distribution as $Q$ increases.

\section{PREDICTIONS FOR THE LHC AND COMPARISON WITH THE HIGGS BOSON SIGNAL}

Several predictions are presented in Ref. [4] for the photon-pair continuum in $p p$ collisions at $\sqrt{s}=14 \mathrm{TeV}$. In this summary, I limit my discussion to differences expected between the spectrum of photon pairs from the Higgs boson signal and from the QCD continuum. The dominant production mechanism for the Higgs boson is gluon fusion, $g g \rightarrow H \rightarrow \gamma \gamma$, and we calculate the signal with the same order of precision in the QCD contributions as for the continuum. We include fixed-order initial state QCD corrections at $O\left(\alpha_{s}^{3}\right)$ (NLO) and resummation at NNLL accuracy. These contributions are coded in the same numerical program used to compute the continuum, and we apply the same cuts (for example, on the values of $p_{T}$ and $\eta$ of each photon) for both the signal and the continuum. Use of one code minimizes potential deviations than can arise from codes with different physics content, parton densities, and numerical implementations. We compute the continuum in the range $128<Q<132 \mathrm{GeV}$ and the signal at a fixed Higgs boson mass $m_{H}=130 \mathrm{GeV}$. We use $\mu_{R}=\mu_{F}=Q$ for the signal and the continuum.

The cross section times branching ratio for the Higgs boson signal is substantially smaller than the continuum rate. We present distributions normalized to the respective 


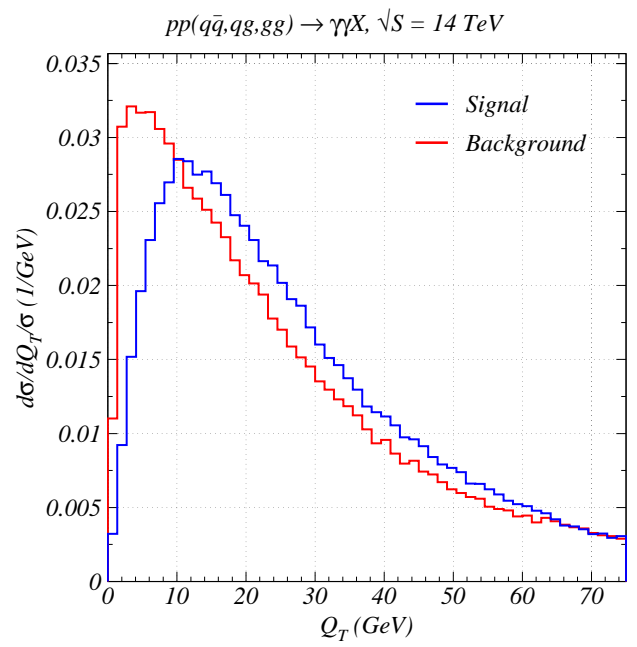

FIGURE 2. Comparison of the normalized transverse momentum distributions for the Higgs boson signal and diphoton continuum at the LHC, both computed at NNLL accuracy. The continuum is calculated for $128<Q<132 \mathrm{GeV}$, and the Higgs boson mass is taken to be $m_{H}=130 \mathrm{GeV}$.

total rates in Fig. 2. The signal and continuum peak at about 12 and $5 \mathrm{GeV}$, respectively. The dominant Sudakov exponent $C_{F}=A_{q \bar{q}}^{(1)}=A_{q g}^{(1)}$ that controls gluon radiation for the $q \bar{q}$ and $q g+\bar{q} g$ initial states is less than $A_{g g}^{(1)}=C_{A}$ that controls the $g g$ case. More gluon radiation in the $g g$ case explains the broader $Q_{T}$ spectrum for the Higgs boson signal. The comparison in Fig. 2 suggests that the signal to background ratio would be enhanced if a cut is made to restrict $Q_{T}>10 \mathrm{GeV}$. Additional differences between the signal and continuum are shown in Ref. [4], including the rapidity difference of the two photons, the scattering angle in the Collins-Soper frame $\tanh \left(\left(y_{1}^{\gamma}-y_{2}^{\gamma}\right) / 2\right)=\cos \theta^{*}$, and the difference between the azimuthal angles of the photons.

\section{ACKNOWLEDGMENTS}

Work in the High Energy Physics Division at Argonne is supported by the U. S. Department of Energy, Division of High Energy Physics, Contract W-31-109-ENG-38.

\section{REFERENCES}

1. E. L. Berger, X.-F. Guo, and J.-W. Qiu, Phys. Rev. Lett. 76, 2234 (1996).

2. P. Aurenche, M. Fontannaz, J. P. Guillet, E. Pilon, and M. Werlen, arXiv:hep-ph/0602133

3. V. M. Abazov et al. (D0 collaboration), Phys.Lett. B639, 1512006.

4. C. Balazs, E. L. Berger, P. Nadolsky, and C. P. Yuan, Phys. Lett. B637, 235 (2006) plus a longer paper in preparation.

5. D. Acosta, et al. (CDF collaboration), Phys. Rev. Lett. 95, 022003 (2005).

6. J. C. Collins, D. E. Soper, and G. Sterman, Nucl. Phys. B250, 199 (1985).

7. E. L. Berger, L. E. Gordon, and M. Klasen, Phys. Rev. D 58,074012 (1998); E. L. Berger, J.-W. Qiu, and X. Zhang, Phys. Rev. D 65, 034006 (2002). 